УКРАЇНСЬКИЙ АНТАРКТИЧНИЙ ЖУРНАЛ

УАЖ, №15, 153-160 (2016)

УДК: 577.112.2:612.128

\title{
THE INFLUENCE OF COLLAGEN FRAGMENTS EXTRACTED FROM THE ANTARCTIC REGION FISH SCALES ON OBESITY DEVELOPMENT
}

\author{
O. Yu. Nagirniak, N. G. Raksha, O. M. Savchuk, L. I. Ostapchenko \\ Educational and Scientific Center "Institute of Biology and Medicine" Taras Shevchenko National \\ University of Kyiv, 64/13, Volodymyrska Str., Kyiv,01601, Ukraine, e-mail:nkudina@ukr.net
}

\begin{abstract}
Collagen is the most abundant protein in vertebrates, which constitutes about $30 \%$ of the total proteins and has been widely used in food, cosmetic, biomedical, and pharmaceutical industries. The conventional source of collagen is from bovine and pig. However, the outbreak of prion diseases, such as bovine spongiform encephalopathy, has resulted in anxiety among users of collagen derived from these land animals. Thus, there is need to find an alternative source of collagen. Fish is one of the candidate as such alternative sources because fish is unlikely to be associated with prion diseases. In addition, their skin, bones, scales, are rich in collagen type I. A simple method has been used for the isolation of collagen from the Antarctic region fish scales. At first, the scales were solublized using acetic acid containing EDTA. Then the solublized collagen was precipitated by using $\mathrm{NaCl}$. The results of sodium dodecyl sulfate polyacrylamide gel electrophoresis confirmed the purity of extracted collagen and showed that obtained collagen was composed of two different $\alpha$-chains ( $\alpha 1$ and $\alpha 2$ ), which were in accordance with the type I collagen. $\beta$ component was also found in lesser amounts in this collagen samples. Next, collagen samples were hydrolyzed with pepsin at $37^{\circ} \mathrm{C}$ for $24 \mathrm{~h}$. According to obtained results collagen was cleaved into low-molecular weight fragments and peptides with molecular weight ranging from 26 to $2 \mathrm{kDa}$. The effect of collagen fragments on obesity development was investigated using animal model of obesity. For this purpose, the rats were fed with a high-calorie diet, which consisted of a standard meal $(60 \%)$, pork fat $(10 \%)$, eggs $(10 \%)$, sugar $(9 \%)$, peanuts $(5 \%)$, dry milk $(5 \%)$ and sunflower oil $(1 \%)$ for four weeks. After that the animals were orally administrated with marine collagen fragments extracted from the Antarctic region fish scales each second day for five consecutive weeks. It was shown that treatment with collagen fragments led to decrease of body weight and body mass index of the animals, which were on high-calorie diet (HCD). Found changes could be result of decrease of food intake and increase of water intake in compare with the rats in HCD group.
\end{abstract}

Key words: Antarctic fishes, scales, collagen fragments, obesity

\section{Introduction}

Diseases caused by metabolic dysfunction such as diabete and obesity have serious effect on human's health. Obesity is a chronic disease of multifactorial origin that develops from the interaction of social, behavioral, psychological, metabolic, cellular, and molecular factors. Obesity is acknowledged as one of the leading health care problems in the world. The prevalence of obesity over the past years has been in constant progression leading the World Health Organization (WHO) to consider it as an epidemic pathology (World Health Organization, 2015). Currently, about 1.5 billion people in the world suffer from this disease. The incidence of obesity has been increasing rapidly in Ukraine. In our country, obesity affects about $15 \%$ of the population and every year the number of new cases of this pathology is 
steadily increasing. Obesity is considered as a top risk factor to develop serious pathologies. It is wellknown that obesity is associated with many chronic diseases such as hyperlipidemia, hypertension, coronary heart disease, type II diabetes, cancers, and other serious diseases (Reaven, 2008). Hence, it's important to find effective functional food or drugs against metabolic dysfunction. Many drugs have been found to treat obesity. But nevertheless on many efforts directed to prevention and treatment of obesity and obesity-related complications, there is no universally satisfactory therapy (Brown et al., 2014). Therefore, the development of new therapeutic approaches and creation new drugs are of great significance.

With marine species comprising approximately one half of the global biodiversity, the ocean offers a wonderful resource for novel compounds, which may serve in improving health of the worldwide population (Grienke et al., 2014). Numerous marine-based compounds have been identified as having diverse biological activities, with some reported to interfere with the pathogenesis of diseases. It has been recognized that bioactive peptides derived from plant and animal food protein can exert of physiological or hormone-like biological activities beyond their nutritional value (Ngo et al., 2012, Takahashi, 2004). Bioactive compounds from marine sources show great potential. Marine bioactive peptides based on their structural properties, amino acid composition have been shown to display a wide range of biological activities including antioxidant, anti-hypertensive, antimicrobial, opioid agonistic, immunomodulatory, prebiotic, mineral binding, anti-thrombotic and hypocholesterolemic effects. Although search for biologically active compounds from marine sources remains a relatively new field, marine organisms represent a vast source of novel active substances and hold a promising future for new drug discoveries because of biodiversity of marine environment. Unfortunately, not all of the sea resources are adequately used. In fact, the skin, bones, scales and residual minced meat of marine animals are considered by-products of the processing industry, which usually cause wastage and pollution. However, these by-products are high in proteins, which offer a resource of functional peptides. In recent years, special attention focused on researches into biopeptides derived from the by-products. They are either thrown back in the sea or retained for production of fishmeal, fish oil, fertilizer, fish silage and animal.

Collagen is one of the key proteins in the body, which represents up to 90 percent of organic bone mass and holds together all living tissue. It provides the infrastructure of the musculoskeletal system making it essential for increased and pain-free mobility. Collagen has been widely used in food, cosmetic, biomedical, and pharmaceutical industries due to its excellent biocompatibility and biodegradability, and weak antigenicity. In recent years, marine originated collagen earned more and more attention due to biological safety, excellent biocompatibility, low antigenicity, high biodegradability, and cell growth potential (Kaewdang et al., 2014, Shoulders et al., 2009). Traditionally, collagen is mainly isolated from the skins of land-based animals, such as cow and pig. At present, because of the risks of bovine spongiform encephalopathy and foot-and-mouth disease associated with land-based animals, the main sources of collagen in many fields are limited. In addition to that, use of livestock as the source of collagen may be limited due to religious reasons or customary practices in some parts of the world. So more and more studies turn to find new safe sources of collagen. Fish is one candidate as such alternative source. There have been many reports of collagen in aquatic animals. Aquatic animals, especially their skin, bones, scales, are rich in collagen type I. In light of the high yield of collagen from fish, fish skin provides an attractive source for collagen. Collagen tissues, including skin, bone and scale account for $30 \%$ of marine fish processing waste. Moreover, using by-products of fish processing as resources for collagen also produced extra profit for the industry. Although therapeutic drugs are currently available for the treatment of obesity, many have adverse side effects or become less effective with time. Currently, nutraceuticals or functional foods, which exhibit not only nutritional benefits but also biological activities, are attractive options for the prevention and management of obesity. Over the past decade, a variety of marine sources have been examined for bioactivity, which have therapeutic potential against several pathologies. A growing number of literatures demonstrated that collagen peptides from marine organisms had a wide spectrum of functional and biological properties. The various multifunctional properties of marine collagen peptides (MCPs) have been described already (Gómez-Guillén et al., 2011, Wang et al., 2014), including anti-microbial activity, anti-ulcer, lipid-lowering effect, wound healing, beneficial effects on skin, and bone. In addition, MCPs may regulate glucose and lipid metabolism, modulate immune function. Researchers have also found that collagen fragments could scavenge hydroxyl radical, superoxide radical, ABTS radical, and DPPH radical effectively, which led to the hypothesis that these peptides could act as a potential candidate for the therapy of diseases associated with oxidative stress, such as obesity.

On that basis, we hypothesize that treatment with MCPs may offer protection for patients with obesity by modulating the pathways involving in obesity pathogenesis. Findings from this study may be of help for potential therapeutic use of marine-derived bioactive peptides in individuals with obesity. 


\section{Materials and Methods}

Marine collagen peptides were prepared from the Antarctic region fish scales. Briefly, wild marine fishes were caught near the island Galindez (geographical coordinates $-65^{\circ} 15^{\prime}$ south latitude, $64^{\circ} 15^{\prime}$ ' west longitude) of Argentine Islands archipelago. The materials were collected by the XVII (March 2012 - April 2013) and XVIII (March 2013 - April 2014) Ukrainian Antarctic expeditions. The scales were washed thoroughly with distilled water, and stored until used.

Extraction of collagen from fish scales was done in two steps (Zhang et al., 2011). Initially, the fish scales were washed twice in $10 \% \mathrm{NaCl}$ solutions (at a ratio dry scales : solution $=1: 10$ ) to remove unnecessary proteins on the surface by stirring the solution for $24 \mathrm{~h}$. Demineralization was achieved with $0.4 \mathrm{M}$ $\mathrm{HClO}_{4}$ solution (at a ratio dry scales : solution $=1: 15$ ) for $90 \mathrm{~min}$. The demineralized scales were washed three times with distilled water. Next collagen from fish scales were extracted with $0.5 \mathrm{M}$ acetic acid containing $5 \mathrm{mM}$ EDTA for $24 \mathrm{~h}$, and the extracts were centrifuged at $10000 \times \mathrm{g}$ for $30 \mathrm{~min}$. The residues were re-extracted with the same solution for $24 \mathrm{~h}$, and these extracts were centrifuged under the same conditions. The supernatants were combined and salted out by adding $\mathrm{NaCl}$ to a final concentration of $0.9 \mathrm{M}$. The precipitated collagens were separated by centrifugation at $10000 \times \mathrm{g}$ for $30 \mathrm{~min}$ and redissolved in $0.5 \mathrm{M}$ acetic acid to precipitate with $\mathrm{NaCl}$ again. The resultant precipitates were dialyzed against distilled water and lyophilized. After being dissolved in $0.1 \mathrm{M}$ acetic acid $(2 \mathrm{mg} / \mathrm{Ml})$, the collagen was enzymatically hydrolyzed by pepsin $(3000 \mathrm{U} / g$ protein $)$ at $37^{\circ} \mathrm{C}$ and for $24 \mathrm{~h}$. The resultant hydrolysate was centrifuged at $26000 \times \mathrm{g}$ and subsequently filtered through ceramic membranes $(200 \mathrm{~mm})$ for purification. The purity of obtained collagen peptide fraction was analyzed by SDS-polyacrylamide gel electrophoresis. SDS-PAGE was performed according to the methods of Laemmli (1970) using $6 \%$ resolving gel. The samples were dissolved in $0.6 \mathrm{M}$ Tris- $\mathrm{HCl}$ buffer $(\mathrm{pH} 6.8)$ which contained $5 \%(\mathrm{w} / \mathrm{v})$ sucrose, $2 \%(\mathrm{w} / \mathrm{v}) \mathrm{SDS}, 5 \%(\mathrm{v} / \mathrm{v})$ $\beta$-mercaptoethanol, and $0.1 \%(\mathrm{w} / \mathrm{v})$ Bromophenol blue. After electrophoresis, gels were visualized with Coomassie Brilliant Blue R-250.

Experiments were carried out on white nonlinear female rats with initial weighing of 135-140 g in compliance with the standards of the Convention of Bioethics of the Council of Europe in 1997, European Convention for the protection of vertebrate animals that used for experimental and other scientific purposes, the general ethical principles of animal experiments approved by first National Congress of Bioethics of Ukraine (September 2001) and other international agreements and national legislation in this field. During the first week the rats were received rats received standard food «Purina rodent chow» and water ad libitum. After a one-week acclimation period, the rats were fed with a high-calorie diet, which consisted of a standard meal $(60 \%)$, pork fat $(10 \%)$, eggs $(10 \%)$, sugar $(9 \%)$, peanuts $(5 \%)$, dry milk $(5 \%)$ and sunflower oil (1\%) (Shen et al., 2010) for four weeks. After that the animals were randomly divided into two. Animals of the first group were on a high-calorie diet. Animals of the second group were also on a high-calorie diet. But each second day the rats were orally administrated with marine collagen peptides for five consecutive weeks. The control animals have been fed with a standard food. In general, the study lasted for ten weeks. During the experimental period, the body weight of each animal was measured every week. Feed and water intake were recorded daily in all animal group.

\section{Discussion}

Collagen has a wide range of applications in pharmaceutical, leather and film industries, in cosmetic and biomedical materials, and as food. But due to the outbreak of bovine spongiform encephalopathy, transmissible spongiform encephalopathy and the foot-and-mouth disease crisis, the uses of collagen and collagen-derived products of land animal origin have become of more concern. Therefore, the alternative sources of collagen, especially from aquatic animals including freshwater and marine fish and mollusks have received increasing attention. During the processes of fish, a great amount of fish scales are dumped, which is a great waste because the scales of fish contain a large amount of collagen. It would be more beneficial and economical to use by-products of fish processing as a source of collagen (Senevirathne et al., 2012).

Thus, the objectives involved were extraction of collagen from fish scale, treating them with enzyme to generate collagen peptides fraction, and evaluate the influence of collagen peptide on obesity development using corresponding animal model.

At first, we extracted and partially characterized the collagen of fish scales for potential utilization. Because the main component of scales is type I collagen, this study was focused on extraction and isolation of collagen type I. Fig. 1 showed the molecular weight pattern of collagen extracted from the Antarctic region fish scales against the high molecular weight markers. The protein patterns were analyzed by $6 \%$ resolving gel. It was found that the major constituents of obtained collagen consisted of $\alpha 1$ - and $\alpha 2$-chains at 
O. Yu. Nagirniak, N. G. Raksha, O. M. Savchuk, L. I. Ostapchenko

THE INFLUENCE OF COLLAGEN FRAGMENTS EXTRACTED FROM THE ANTARCTIC REGION

FISH SCALES ON OBESITY DEVELOPMENT

a ratio of approximately $2: 1$ (Kimura et al., 1987). Based on this fact extracted collagen should most likely be classified as type I collagen. Apart from $\alpha$-chains, collagen also contained high molecular weight components, including $\beta$ - and c-components as well as their cross-linked molecules.

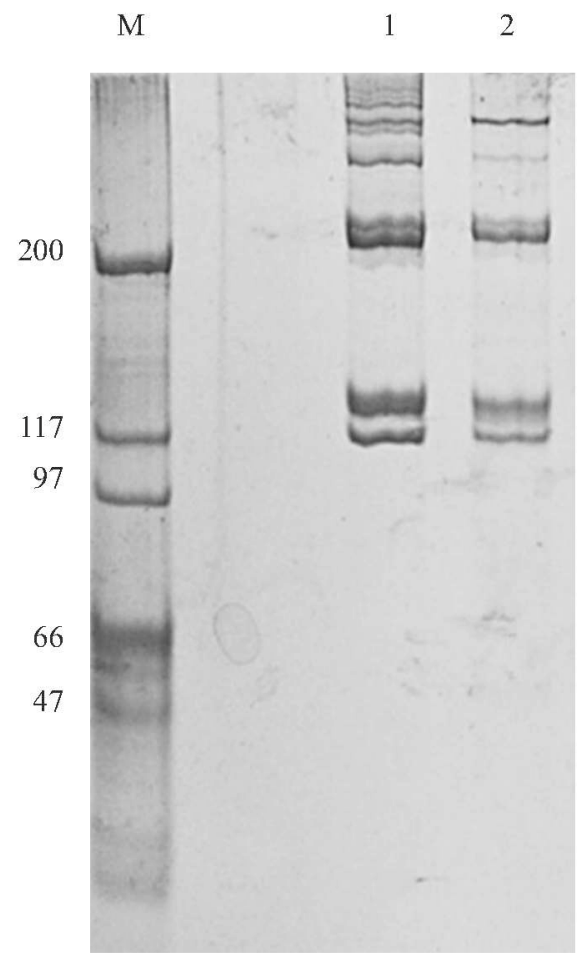

Fig. 1.Electrophoregram of collagen extracted from the Antarctic region fish scales: $\mathrm{M}$ - molecular weight markers $(\mathrm{kDa}) ; 1-2$ - the collagen samples

The prevalence of obesity over the past years has been in constant progression leading the World Health Organization to consider it as an epidemic pathology. Obesity is a chronic disease of multifactorial origin and can be defined as an increase in the accumulation of body fat. Adipose tissue is not only a triglyceride storage organ, but studies have shown the role of white adipose tissue as a producer of certain bioactive substances called adipokines. Adipokines induce the production of reactive oxygen species, generating a process known as oxidative stress, which plays critical roles in the pathogenesis of obesity as well as obesity associated complications. The hypothesis that oxidative stress is causative in the development of metabolic disorders has been supported by different studies where treatments reducing ROS production mediate therapeutic effects. Numerous researches have demonstrated that collagen peptides extracted from marine organisms provide a wide spectrum of biological activities. These bioactive properties or health-enhancing potentials have led to the use them as ingredients of functional foods or pharmaceuticals. It was found that treatment with MPCs improved insulin sensitivity in insulin-resistant individuals and reduced insulin-resistance related metabolic disorders. In addition, MCPs could reduce hyperlipidemia, modulate immune function, and provide antioxidation activity in animal models. These therapeutic effects suggest that MCPs may be used in addition to regular therapy of diabetes and obesity. Taking into account all described above the next goal of present study was to obtain marine collagen peptides and assess whether these peptides could influence the development of obesity.

There are some approaches to produce bioactive peptides from different sources. Among them enzymatic hydrolysis has been found as more suitable method to obtain functionally active peptides. In our experiment collagen samples were hydrolyzed with pepsin at $37^{\circ} \mathrm{C}$ for $24 \mathrm{~h}$. The obtained hydrolysates were analyzed by SDS-PAGE for estimation the degree of collagen hydrolyis. According to obtained results after digestion by pepsin (Fig. 2), collagen was cleaved into some low-molecular weight fragments and peptides with molecular weight ranging from 26 to $2 \mathrm{kDa}$. No high-molecular fragments of collagen were found at electrophoregram. Such result indicated about sufficient of applied method of hydrolysis. 


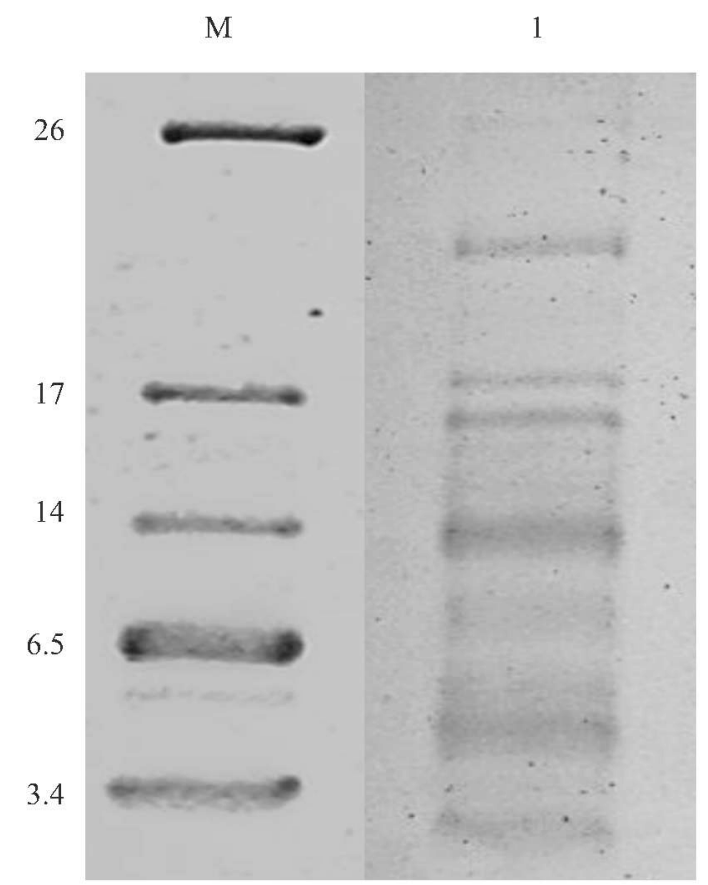

Fig. 2. Electrophoregram of collagen fragments: $\mathrm{M}$ - molecular weight markers $(\mathrm{kDa})$; 1 - the collagen fragments sample

The data shown in Fig. 3 represented the dynamics of body weight increase in the experimental groups throughout all investigated period. The initial weight of the rats in the control group was $183.8 \pm 4.1$ $\mathrm{g}$. A gradual increase of body weight of the control animals was observed during ten weeks of the experiment. On the last week of study the weight of the control animals was $386.5 \pm 2.7 \mathrm{~g}$, which was on $202 \mathrm{~g}$ more than initial value. It was found that in the rats that were on high-calorie diet the body weight at the end of the investigation period was on $272 \mathrm{~g}$ higher than their initial weight $(171.1 \pm 3.2 \mathrm{~g})$. Body weight of rats which were orally administrated with MCPs was $172.4 \pm 2.9 \mathrm{~g}$ and increased on $229 \mathrm{~g}$.

Summarizing, the body weight of control animals after ten weeks of experiments increased on $110.2 \%$ in compare with the initial weight. Initial weight of the animals on high-calorie diet and animals treated with MCPs increased on $161.3 \%$ and $132.7 \%$, respectively (Fig. 4).

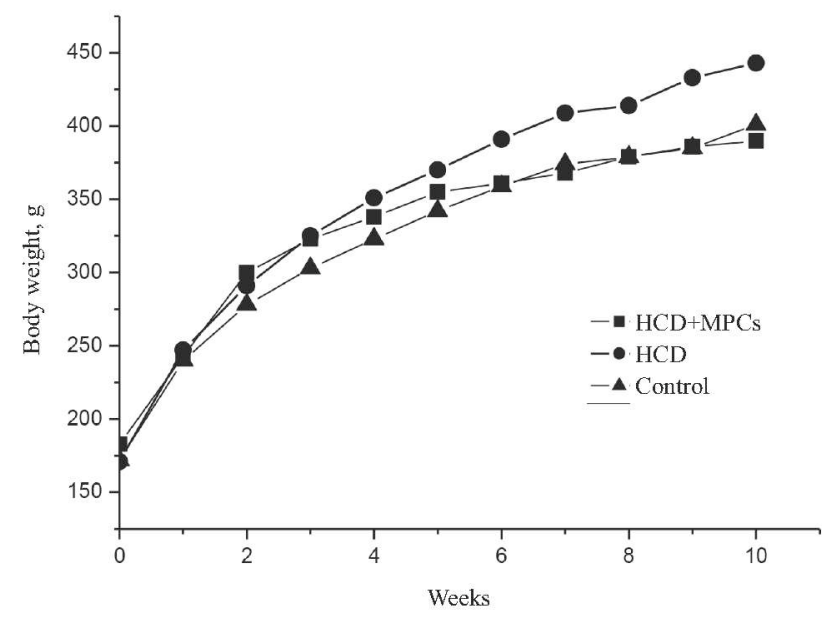

Fig. 3. Body weight of the rats in the "HCD" and "HCD+MPCs" groups $(M \pm m ; n=10)$ 


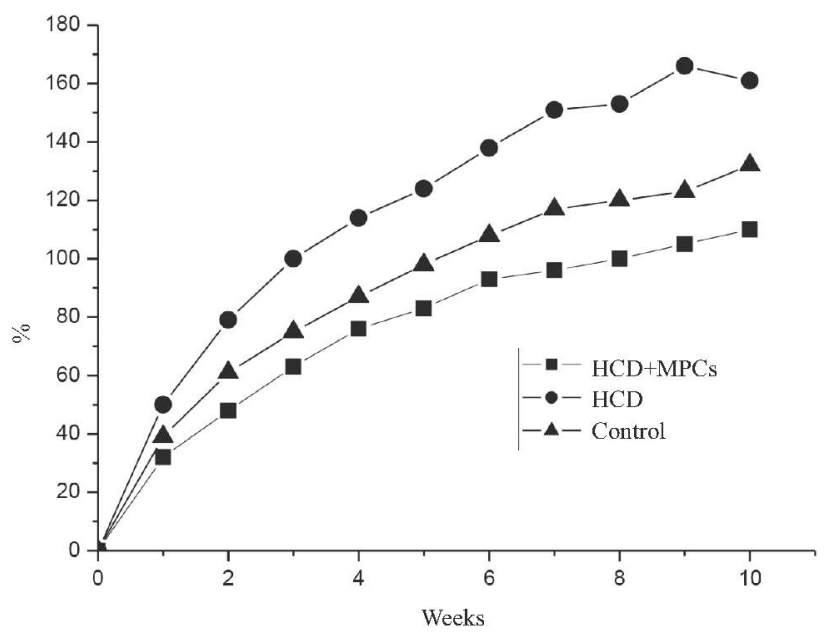

Fig. 4. The dynamics of body mass increase of the rats in the "HCD" and "HCD $+M P C s "$ groups $(M \pm m ; n=10)$

It has been recently recognized that protein is the most satiating macronutrient. Moderate intake of protein diet plays a crucial role in body weight loss and weight maintenance. The proposed mechanisms for weight loss include increased satiety and thermogenesis, accretion of fat free mass and lowering food intake, resulting in decreased body weight (Seale, 2009). Collagen peptides have been shown to have a higher satiating effect in comparison to other proteins. A randomized experimental study has found that those individuals who consumed a breakfast in which 10 to 25 percent of the energy was provided by collagen peptides, or collagen peptides enriched with tryptophan, reduced their energy intake by around 20 percent during the following lunch, demonstrating 30 to 50 percent more satiation than casein, soy and whey.

It should be noted that increased of body weight, by itself, cannot be considered as direct confirmation of obesity. One of the most commonly used ways of estimating whether an individual person is overweight or obese is calculating of body mass index (BMI). BMI is defined as weight in kilograms divided by height in meters squared. In general, the higher the number, the more body fat a person has. Therefore, for conformation the obesity development we calculated BMI for all experimental groups. According to obtained results (Table) BMI of the control animals after ten weeks of experiment was $0.7 \mathrm{~g} / \mathrm{cm}^{2}$, which was within the range for rats of corresponding age (Novelli et al., 2007). We have revealed an increase of BMI of the animals on high-calorie diet by 1.12 times compared with the control level after ten weeks of experiments. Studies shown that BMI in the MPCs treatment group also increased, but this value was lower than those for on high-calorie diet.

Table

General characteristics of the rats which were on a high-calorie diet (HCD) and the rats orally administrated with collagen fragments from the Antarctic region fish scales (HCD $+\mathrm{MPCs})(\mathrm{M} \pm \mathrm{m}, \mathrm{n}=10)$

\begin{tabular}{|l|c|c|c|}
\hline & Control & HCD + MPCs & HCD \\
\hline BMI $\left(\mathrm{g} / \mathrm{cm}^{2}\right)$ & 0.7 & 0.74 & 0,78 \\
\hline Food intake $(\mathrm{g} /$ day $)$ & $28 \pm 1.2$ & $25 \pm 1.3$ & $31 \pm 1.5^{*}$ \\
\hline Water intake $(\mathrm{ml} /$ day) & $38 \pm 2.1$ & $34 \pm 1.9$ & $32 \pm 2.3^{*}$ \\
\hline
\end{tabular}

${ }^{*} \mathrm{p}<0.05$ significantly different from the control group

At the next stage of our work we studied food intake by rats of all experimental groups. Obtained changes were shown in Table. According to our results, the control group of animals consumed an average of $28 \pm 1.2 \mathrm{~g}$ standard food per day. The rats which were on high-calorie diet ate approximately 
$31 \mathrm{~g}$ of food. It was in 1.2 times higher than ate rats of the control group. These results suggest the development of obesity in rats on high-calorie diet. Increased body weight of the rats of this is probably associated with the accumulation of adipose tissue. This accumulation may be the result of an imbalance between the amount of energy consumed by rats and the amount of energy spent, because alongside with the increasing amount of consumed feed by the animals of the experimental group, the amount of energy received was increased either due to high caloric content of food. Based on these data, it can be argued that the development of obesity in rats of experimental group arises from the phenomenon of hyperphagia. We found a slight inhibition effect of MPCs treatment on food intake. Animals, which were orally administrated with MPCs consumed about $25 \pm 1.3 \mathrm{~g}$ high-calorie feed per day. This value did not differ significantly from the control animals but was lower in compare with the animals of HCD group.

Such result partly could be explained from the position of MPCs influence on cholecystokinin - a biomarker associated with satiety, which inhibit food intake. It has been established that low molecular weight peptides $(1-1.5 \mathrm{kDa})$ could be an effective agent for stimulation of cholecystokinin release in STC1 cells (Moran, 2000). Thus, these peptides are suggested as a promising functional food against obesity via regulation of cholecystokinin release.

In addition, changes in water intake were analyzed during the experiment. In the course of these studies it was not found significant differences between group of animals on high-calorie diet and those administrated with MPCs compared with the control. The value of water intake was $38 \pm 2.1,32 \pm 2.3$ and $34 \pm 1.9 \mathrm{ml}$ per day for the control group, animals, which were on high-calorie diet and animals treated with MPCs, respectively.

Thus, our results demonstrated the ability of collagen fragments extracted from Antarctic region fish scales to influence the development of obesity, in particular, to reduce the amount of consumed food, which were accompanied by decrease of body mass index and body weight in compare with those for animals, which were on high-calorie diet.

This research was supported by National Antarctic Scientific Center of Ukraine Ministry of Education and Science of Ukraine.

\section{References}

1. Brown W., Bays H., Bray G. JCL Round table: Clinical managementof individuals with obesity // Journal of Clinical Lipidology. - 2014. - № 8. - P. 237-248.

2. Gómez-Guillén M. C., Giménez B., López-Caballero M. E. et al. Functional and bioactive properties of collagen and gelatin from alternative sources: A review. Food Hydrocolloids. - 2011. - № 25. - P. 1813-1827.

3. Grienke U., Silke J., Tasdemir D. Bioactive compounds from marine mussels and their effects on human health// Food Chemistry. - 2014. - № 142. - P. 48-60.

4. Kaewdang O., Benjakul S., Kaewmanee T. et al. Characteristics of collagens from the swim bladders of yellowfin tuna (Thunnusalbacares) // Food Chemistry. - 2014 - 15(155). - P. 264-270.

5. Kimura S., Ohno Y., Miyauchi Y. et al. Fish Skin Type I Collagen: Wide Distribution of an a3 Subunit in Teleosts // Comparative Biochemistry and Physiology. - 1987. - Vol. 88B, № 1. - P. 27-34.

6. Laemmli K. Cleavageofstructuralproteinsduringtheassemblyoftheheadofbacteriophage T4 / Laemmli K. // Nature. - 1970. - Vol.227, №1 - P. 680-685.

7. Moran T. H. Cholecystokinin and satiety: current perspectives // Nutrition. -2000 . - 16(10). - P. 858-865.

8. Ngo D-H., Vo T-S., Ngo D-N. et al. Biological activities and potential health benefits of bioactive peptides derived from marine organisms // International Journal of Biological Macromolecules. - 2012. - № 51. P. $378-383$.

9. Novelli E., Diniz Y., Galhardi C. Anthropometrical parametersand markersof obesityin rats // LaboratoryAnimals. - 2007. - № 41. - P. 111-119.

10. Reaven G. M. Insulin resistance: the link between obesity and cardiovascular disease // Endocrinol Metab Clin North Am. - 2008. - 37. - P. 581-601.

11. Seale P., Lazar M. Brown Fat in Humans: Turning up the Heat on Obesity // Diabetes. - 2009. - Vol. 58, №7. - P. 1482-1484.

12. Senevirathne M., Kim S. K. Utilization of seafood processing by-products: Medicinal applications // Advances in Food and Nutrition Research. -2012. - № 65. - P. 495-512.

13. Shen X., Tang Q., Huang J. et al. Vitamin E regulates adipocytokine expression in a rat model of dietary-induced obesity // Experimental Biologyand Medicine. - 2010. - № 235. - P. 47-51 
O. Yu. Nagirniak, N. G. Raksha, O. M. Savchuk, L. I. Ostapchenko THE INFLUENCE OF COLLAGEN FRAGMENTS EXTRACTED FROM THE ANTARCTIC REGION FISH SCALES ON OBESITY DEVELOPMENT

14. Shoulders M. D., Raines R.T. Collagen structure and stability // Annual Review of Biochemistry. - 2009. №78. - P.929-958.

15. Takahashi K. Translational medicine in fish-derived peptides: from fish endocrinology to human physiology and diseases // Endocr J. - 2004. - №51. - P. 1-17.

16. Wang H., Fu Z., Han C. The Potential Applications of Marine Bioactives Against Diabetes and Obesity // American Journal of Marine Science. - 2014. - Vol. 2, № 1. - P. 1-8.

17. World Health Organization. Obesity and overweight [Електронний pecypc] /. World Health Organization // WHO Fact sheet. - 2015. - Режим доступу до ресурсу: http://www.who.int/mediacentre/factsheets/fs311/en/.

18. Zhang F., Wang A., Li Z. et al. Preparation and Characterisation of Collagen from Freshwater Fish Scales // Food and Nutrition Sciences. - 2011. - № 2. - P. 818-823 